\section{Towards complete endoscopic staging of the mediastinum?}

R. Rintoul

Papworth Hospital, Cambridge, UK

\section{Introduction}

Accurate staging of the mediastinum is a critical part of the management for newly diagnosed cases of lung cancer. It is well recognised in non small cell lung cancer (NSCLC) that stage at presentation is the main determinant of survival. Furthermore, the degree of spread will determine the most appropriate and optimal treatment strategy. NSCLC usually metastasises initially via the lymphatic system to locoregional lymph nodes (hilar and mediastinal) and subsequently to distant organs by haematogenous spread.

\section{Surgical approaches to staging}

The traditional surgical approach to mediastinal staging is to perform a mediastinosocopy. A standard cervical mediastinoscopy, when performed by an experienced thoracic surgeon should allow access to the right and left paratracheal lymph node stations as well as to the uppermost part of the subcarinal lymph node group (Fig. 1a). However, it is well recognised that mediastinoscopy is not always a straightforward procedure and optimal yield is related to the experience of the operator. Less experienced surgeons may not feel confident about fully exploring the mediastinum for fear of damaging mediastinal structures. Pooled data showed that mediastinoscopy had a sensitivity of $81 \%$ and specificity of $100 \%$ [1]. Although many case series report a higher sensitivity than this, it is probably an accurate reflection of average clinical practice. A recent study showed that only $40 \%$ of mediastinoscopies are performed adequately [2].

Although a mediastinoscopy has been long regarded as the goldstandard for mediastinal staging it is important to realise that no one surgical procedure provides access to all mediastinal lymph node stations. When staging NSCLC it is important to appreciate the relative likelihood and distribution of mediastinal lymph node metastases. In general the smaller and more peripherally situated a lung cancer, the less likely it is to have metastasised to mediastinal lymph nodes. Histological type also influences the risk of metastasis. In general, squamous and bronchoalveolar subtypes are less prone to metastasise than adenocarcinoma. Because of the anatomy of the lymphatic drainage system, the lobar location of a lung cancer will influence the likely site of nodal metastases and this should be considered during staging investigations [3]. Right upper lobe tumours usually spread to the right paratracheal lymph nodes (levels $2 \mathrm{R}$ and $4 \mathrm{R}$ ). Right middle lobe

Correspondence: Dr Robert C Rintoul · Consultant Respiratory Physician · Department of Thoracic Oncology · Papworth Hospital · Cambridge CB3 8RE - UK . Phone: +441480364342 · Fax: +441480364331 · E-mail: robert.rintoul@papworth.nhs.uk

Bibliography: Endoscopy 2006; 38 (S1): S110-S113 @ Georg Thieme Verlag KG Stuttgart · New York · ISSN 0013-726X · DOI 10.1055/s-2006-946669 and lower lobe tumours from either side commonly spread to the subcarinal nodes (level 7) initially. Left lower lobe tumours are unusual in that they have a high risk of metastasising to contralateral right paratracheal lymph nodes. On the contrary, left upper lobe tumours which have a propensity to metastasise to aortopulmonary window (level 5) and para-aortic (level 6) lymph nodes initially, may still be fully resectable despite being stage IIIA. If nodal spread is confined to levels 5 and 6 they have a relatively better prognosis than other $\mathrm{N} 2$ nodal subsets of stage IIIA.

These well recognised patterns of disease have historically influenced the surgical approach to mediastinal staging. A standard cervical mediastinoscopy allows access to levels $2 \mathrm{R}, 2 \mathrm{~L}, 4 \mathrm{R}, 4 \mathrm{~L}$ and the upper anterior part of level 7. Lymph node groups 5 and 6 lying in the aortopulmonary window and para-aortic area respectively may be accessed by a left anterior mediastinotomy. With the advent of video assisted thoracoscopic (VATS) procedures, a left VATS permits access to the whole of the subcarinal area and to the posterior-inferior mediastinum including lymph node levels 8 and 9 .

During the last two decades, developments in imaging technology, initially computed tomography (CT), then positron emission tomography (PET) and most recently PET-CT have shaped the staging algorithm for non-small cell lung cancer (NSCLC). However, informative as these techniques are, there strengths and weaknesses must be fully appreciated in order to avoid over-reliance on their results. Pooled data on CT and PET scanning showed that the sensitivity and specificity for predicting malignant involvement of mediastinal lymph nodes were $60 \%$ and $81 \%$ and $84 \%$ and $89 \%$ respectively [4]. Recent data from PozoRodriguez et al have shown that the negative predictive value of combined CT and PET can be as high as $98 \%$. While these data suggest that a negative PET-CT is sufficiently accurate to obviate the need for other pre-thoracotomy staging investigations, the same can not be said for patients with a positive scan. The falsepositive rate of CT and PET is sufficiently high to demand pathological confirmation in order to prevent patients being over-staged on radiological investigations alone and being denied potentially curative treatment [6].

\section{Endoscopic staging procedures}

\section{Endoscopic ultrasound}

Endoscopic ultrasound (EUS), which has been used by gastroenterologists for a number of years for oesophageal staging, is increasingly being used to examine the mediastinum in lung cancer. With the advent of curvilinear echoendoscopes which allow real-time imaging of fine needle aspiration (FNA), it is possible to sample mediastinal lymph nodes. The route of the oesophagus dictates the lymph node stations that are readily accessible. Lymph nodes located in the aortopulmonary window (medial to the ligamentum arteriosum - level $4 \mathrm{~L}$ ), subcarinal space (level 7), paraoesophageal region (level 8) and inferior pulmonary ligament (level 9) should be amenable to biopsy (Fig. 1b). Furthermore, EUS can be used to examine (and biopsy) lesions in the left adrenal gland and the left lobe of the liver. The air-filled trachea lying anterior to the oesophagus usually prevents imaging 

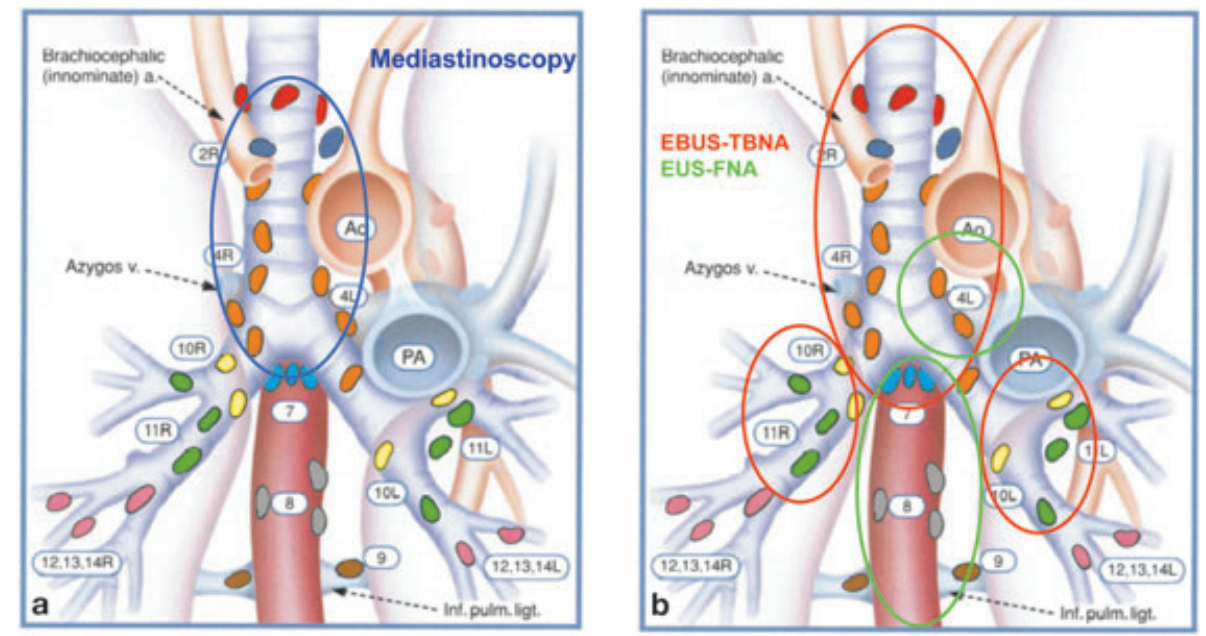

Fig. 1 Figure 1a shows the lymph node stations that can normally be accessed by a standard cervical mediastinosocopy. Figure 1b shows the lymph node stations that can usually be accessed by endobronchial ultrasound (EBUS-TBNA) and endoscopic ultrasound fine needle aspiration (EUS-FNA). The diagrams of the lymph node stations are after Mountain CF and Dresler CM. Regional lymph node classification for lung cancer staging. Chest 1997; 111:17181723.

of the hilar and paratracheal lymph nodes although occasionally left paratracheal lymph nodes can be seen. In expert hands the sensitivity of EUS-FNA for detection of malignant lymph nodes is around $90-95 \%[1,7,8]$. However, the majority of these studies are retrospective and report on lymph nodes $>1 \mathrm{~cm}$ in short-axis diameter as reported by CT scanning. This suggests that there may be a selection bias present in these studies. Annema et al., have recently reported the results of a large prospective study on the use of routine EUS-FNA in patients with known or suspected lung cancer scheduled to undergo surgical staging procedures [9]. They found that EUS-FNA prevented $70 \%$ of surgical procedures on account of upstaging. These findings support those of two other recent studies which both showed that EUS can obviate the need for surgical staging procedures $[10,11]$. However, it should be noted that in all of these studies the inclusion criteria stipulated lymph node size greater than $10 \mathrm{~mm}$ on CT and in Annema's study the size range was $13-77 \mathrm{~mm}$ with a mean of $24 \mathrm{~mm}$ [9]. This therefore raises the question as to the role of EUS in lung cancer patients with a 'CT-node negative' mediastinum. Two groups have investigated this scenario. Wallace et al., [12] showed that 17 of 69 (25\%) patients with lung cancer had unsuspected disease on CT which was detected by EUS-FNA. More recently, LeBlanc et al., [13] found that EUS precluded inappropriate surgery in $12 \%$ of patients without mediastinal lymphadenopathy, based on the presence of N2 or N3 node positive disease. Although neither of these studies incorporated PET scanning their results do indicate that EUS probably has a significant role to play in staging of a 'normal' mediastinum. Interestingly, LeBlanc et al. noted that EUS detected more occult metastases in cases of hilar and lower lobe tumours than upper lobes [13]. Based on the known patterns of lymphatic drainage described above, this is not surprising.

Overall, the emerging data on EUS-FNA shows that it has a major role to play in the diagnosis and staging of lung cancer. Furthermore, it is complimentary to, rather than competitive with, mediastinoscopy. Confirmation of this has come in two recent studies which have clearly shown that EUS-FNA can add to the findings of mediastinoscopy $[14,15]$.

\section{Transbronchial needle aspiration}

Transbronchial needle aspiration (TBNA) of lymph nodes and parabronchial lesions was first described by Wang in 1983 [16] and although it is an established bronchoscopic technique it remains underutilised $[17,18]$. The main reasons for this are its variable biopsy yield and long learning curve. Until recently TBNA has been limited to 'blind' needle puncture guided by static CT scans. This technique is highly operator dependent and reported sensitivity varies between $20 \%$ and $80 \%[16,19,20]$. Understandably the larger the target lymph node the higher the chance of success and higher yields are obtained from certain lymph node stations such as level 4R (right paratracheal) and level 7 (subcarina) [21]. Yield is also improved with at least seven passes of the TBNA needle and on site cytopathology [22]. More recently Herth et al. [23] have demonstrated that a mechanical radial ultrasound mini-probe introduced through the working channel of a flexible bronchoscope, can be used to localise target lymph nodes prior to sequential blind biopsy using a standard bronchoscope and TBNA needle. By this method, sampling sensitivity rose to $86 \%$. While this technique has improved TBNA yields, it does not allow ultrasound-guided control of needle puncture and inadvertent puncture of vascular structures remains a risk, albeit a small one.

Most recently endobronchial ultrasound guided transbronchial needle aspiration (EBUS-TBNA) has been described. This utilises a curvilinear echoendoscope similar to those used for EUS-FNA which allows real-time visualisation of needle puncture. Several groups have now reported their initial results using this novel device with sensitivities in the range of $85-95 \%$ [24-28]. Using this device it has been shown that it is possible to successfully biopsy lymph nodes down to $7 \mathrm{~mm}$ in diameter from the upper mediastinum lymph node stations 2,3 , and 4, subcarina (station 7) and hilar stations (10 and 11) (Fig. 1b). An endobronchial approach allows wider access to upper mediastinal and hilar nodal stations than does a mediastinoscopy. The deeper regions of the subcarinal space are easily examined from the right and left main bronchi and the hilar lymph nodes (station 10 and 11) can routinely be identified. Like EUS, EBUS-TBNA can be easily performed under conscious sedation as a day case procedure and has a negligible complication rate. 
Combined endobronchial and endoscopic ultrasound for staging the mediastinum

The recent advances in endobronchial ultrasound guided TBNA now make it possible to consider using minimally invasive techniques to stage almost the whole mediastinum. In conjunction with endoscopic ultrasound it should be possible to examine the upper mediastinal, hilar and posterior-inferior lymph node stations (Fig. 1b). The only lymph node groups that are not accessible to either approach are station 5 (aortopulmonary window lateral to the ligamentum arteriosum and station 6 (para-aortic).

Two groups have reported their preliminary experience of this approach. In their paper reporting their initial experience of the EBUS-TBNA bronchoscope, Rintoul et al., [27] reported on a subset of 7 patients on whom they had performed both EUS-FNA and EBUS-TBNA. They found that the addition of EUS added extra information to the examinations in all cases. The procedures were well tolerated in all cases and the mean time for the combined procedures was 45 minutes. Subsequently Vilmann et al., [29] compared findings by EUS-FNA and EBUS-TBNA in 33 patients. Again they observed that EUS and EBUS gave complimentary information - in a number of cases EBUS-TBNA gave specific cancer diagnoses in lymph node groups that had not been obtained by EUS and vice versa. This most likely relates to the fact that the approach to certain nodal stations such as the subcarina is different by each method and resultant needle biopsies sample different nodes and parts of nodes. In this series the accuracy of EUSFNA and EBUS-TBNA, in combination, for the diagnosis of mediastinal cancer was $100 \%$.

\section{Mediastinal re-staging after induction chemotherapy for} NSCLC

The use of neo-adjuvant chemotherapy to downstage NSCLC prior to surgery has focussed attention upon the problems inherent in re-staging of the mediastinum. It is recognised that post-chemotherapy imaging modalities such as CT and PET loose sensitivity and specificity. Re-mediastinoscopy is also more technically challenging due to adhesions and fibrotic change consequent upon the initial surgery and chemotherapy [30,31]. As a result diagnostic accuracy is lower with more incomplete procedures and complications [32]. The minimally invasive approaches of EBUS-TBNA and EUS-FNA offer new approaches to staging and re-staging the mediastinum around neo-adjuvant chemotherapy. To date there is only one small study using EUS that has examined its role in re-staging the mediastinum. Annema et al., [33] reported on 18 patients who underwent EUS-FNA following chemotherapy for stage IIIA-N2 disease. They reported a diagnostic accuracy of $83 \%$ for correct prediction of $\mathrm{N} 2$ nodal status. EUS-FNA negative cases were confirmed by surgical exploration. This result is similar to published results using re-mediastinoscopy as a staging tool. It is likely that if EBUS-TBNA and EUSFNA were combined in this situation even better results might be possible. It is interesting to note that in 2 of the 3 false negative cases in Annema's series, disease positive lymph nodes were found in the paratracheal regions - an area difficult to routinely sample by EUS.
Pros and cons of minimally invasive mediastinal staging versus surgical staging

EBUS and EUS clearly offer great potential for staging the mediastinum in a minimally invasive manner. From the patients point of view they are far less invasive than surgery and can be performed as a day case under conscious sedation and local anaesthetic without the need for an in-patient stay and general anaesthetic. From the clinicians point of view they are quick to undertake and in combination offer a wider access to different mediastinal stations than mediastinoscopy or thoracoscopy alone. However, while it is likely that these techniques will replace the need for surgical staging procedures to a large extent, it is unlikely that they will replace them entirely. At present there have been no controlled trials comparing the accuracy of EBUS and EUS with mediastinoscopy or thoracoscopy. Future studies are required to define the best use and combination of these techniques. There will be times when it is important to obtain large biopsies rather than cytological FNA samples. While cytological specimens are usually sufficient to diagnose carcinoma, the same can not be said of lymphoma or mesothelioma. In our practice if mediastinal biopsy is required to diagnose or stage either of these conditions we proceed directly to surgery. We have also found on occasion that cytological samples from large necrotic or very hard scirrhous lymph nodes are inadequate for diagnosis and surgical biopsy is necessary. Larger 19-gauge ‘histology' EUS needles are under development which may help in certain situations but it is unlikely that needles of this size will be possible for EBUS given size restrictions imposed by anatomy.

The reduction in the number of mediastinoscopies being performed will also have implications on surgical training and experience [34]. As stated earlier, a mediastinoscopy should not be regarded as a straightforward procedure and its accuracy like any invasive procedure is related to the expertise of the clinician performing it. There is an understandable concern in surgical circles that if trainee surgeons are not exposed to sufficient numbers of the 'easier' cases they will not be in a position to manage a challenging one.

\section{NSCLC staging algorithm of the future (Fig. 2)}

In summary, the standard of care for mediastinal staging is changing. As techniques such as PET-CT and EBUS-TBNA and EUS-FNA become more widespread the diagnostic and staging algorithm for lung cancer will change. In future, the staging algorithm for NSCLC might start with a contrast CT of the neck, chest and abdomen and then for patients who appear to have radically treatable disease progress to a PET-CT scan with brain imaging as clinically indicated. Based on this, diagnostic and staging tissue biopsy procedures would be performed with the ethos being to diagnose and stage to the highest stage in as few steps as possible. This might involve an initial combination of bronchoscopy and/or EBUS and/or EUS. Surgical staging procedures might be held in reserve when other staging studies are equivocal or when the need for larger amounts of tissue is clinically indicated. In the situation where re-staging following neo-adjuvant therapy is indicated initial staging using a combination of EBUS and EUS would allow surgical staging to be reserved for re-staging if 
CT Scan (Neck, chest, abdomen)

for suspected NSCLC

(Candidate for radical treatment

No clear evidence of stage IIIB or IV disease)

\section{CT-PET}

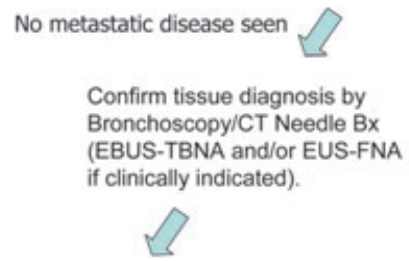

Thoracotomy

Treatment dependent upon results

Fig. 2 Potential NSCLC staging algorithm of the future for patients with potentially radically treatable disease incorporating early use of PET-CT and targeted biopsy of disease using minimally invasive approaches whenever possible.

necessary although it is likely that EBUS and EUS will have a major role to play here too.

\section{References}

${ }^{1}$ Toloza EM, Harpole L, Detterbeck F et al. Invasive staging of non-small cell lung cancer: A review of the current evidence. Chest 2003; 123: $157 S-166 S$

2 Smulders SA, Smeenk FWJM, Janssen-Heijnen MLG et al. Surgical mediastinal staging in daily practice. Lung Cancer 2005; 47: 243-251

${ }^{3}$ Libshitz HE, McKenna RJ, Mountain CF. Patterns of mediastinal metastases in bronchogenic carcinoma. Chest 1986; 90: 229-232

${ }^{4}$ Toloza EM, Harpole L, McCrory DC et al. Noninvasive staging of nonsmall cell lung cancer: a review of the current evidence. Chest 2003; 123: $137 \mathrm{~S}-146 \mathrm{~S}$

${ }^{5}$ Pozo-Rodriguez F, Marin de Nicolas JL, Sanchez-Nistal MA et al. Accuracy of helical CT and FDG-PET for identifying lymph node mediastinal metastases in potentially resectable non-small cell lung cancer. J Clin Oncol 2005; 23: 8357-8361

${ }^{6}$ Pieterman RM, van Putten JW, Meuzelaar JJ et al. Preoperative staging of non-small-cell lung cancer with positron emission tomography. $\mathrm{N}$ Engl J Med 2005; 343: 254-261

${ }^{7}$ Wallace MB, Fritscher-Ravens A, Savides TJ. Endoscopic Ultrasound for the staging of Non-small cell lung cancer. Endoscopy 2003; 35: 606 610

${ }^{8}$ Fritscher-Ravens A. Endoscopic ultrasound evaluation in the diagnosis and staging of lung cancer. Lung Cancer 2003; 41: 259-267

${ }^{9}$ Annema JT, Versteegh MI, Veselic M. Endoscopic ultrasound-guided fine-needle aspiration in the diagnosis and staging of lung cancer and its impact on surgical staging. J Clin Oncol. 2005; 23: 8357-8361

${ }^{10}$ Larsen SS, Krasnik M, Vilmann P et al. Endoscopic ultrasound guided biopsy of mediastinal lesions has a major impact on patient management. Thorax 2002; 57: 98-103

${ }^{11}$ Savides TJ, Perricone A. Impact of EUS-guided FNA of enlarged mediastinal lymph nodes on subsequent thoracic surgery rates. Gastrointest Endosc 2004; 60: 340-346

12 Wallace MB, Raven el J, Block MI et al. Endoscopic ultrasound in lung cancer patients with a normal mediastinum on computed tomography. Ann Thorac Surg 2004; 77: $1763-1768$

${ }^{13}$ LeBlanc JK, Devereaux BM, Imperiale TF et al. Endoscopic ultrasound in non-small cell lung cancer and negative mediastinum on computed tomography. Am J Respir Crit Care Med 2004; 171: 177 - 182

${ }^{14}$ Eloubeidi MA, Tamhane A, Chen VK et al. Endoscopic ultrasound-guided fine-needle aspiration in patients with non small cell lung cancer and prior negative mediastinoscopy. Ann Thorac Surg 2005; 80: $1231-1239$
${ }^{15}$ Annema JT, Versteegh MI, Veselic M et al. Endoscopic ultrasound added to mediastinoscopy improves preoperative staging of lung cancer. JAMA 2005; 294: 931 - 936

${ }^{16}$ Wang KP, Brower R, Haponik EF et al. Flexible transbronchial needle aspiration for staging of bronchogenic carcinoma. Chest 1983; 84: $571-576$

${ }^{17}$ Smyth CM, Stead RJ. Survey of flexible fibreoptic bronchoscopy in the United Kingdom. Eur Resp J 2002; 19: 458 - 463

18 Pastis NJ, Nietert PJ, Silvestri GA. Variation in training for interventional pulmonary procedures among US pulmonary/critical care fellowships: a survey of fellowship directors. Chest 2005; 127: 1614-1621

${ }^{19}$ Rong F, Cui B. CT scan directed transbronchial needle aspiration biopsy for mediastinal nodes. Chest 1998; 114: 36-39

${ }^{20}$ Gasparini S, Zuccatosta L, De Nictolis M. Transbronchial needle aspiration of mediastinal lesions. Monaldi Arch Chest Dis 2000; 55: 29- 32

${ }^{21}$ Harrow EM, Abi-Saleh W, Blum J et al. The utility of transbronchial needle aspiration in the staging of bronchogenic carcinoma. Am J Resp Crit Care Med 2000; 161: 601 - 607

${ }^{22}$ Chin RJr, McCain TW, Lucia MA et al. Transbronchial needle aspiration in diagnosing and staging lung cancer: how many aspirates are needed? Am J Respir Crit Care Med 2002; 166: 377-381

${ }^{23}$ Herth FJ, Becker HD, Ernst A. Ultrasound-guided transbronchial needle aspiration: an experience in 242 patients. Chest 2003; 123: $604-$ 607

${ }^{24}$ Krasnik M, Vilmann P, Larsen SS, Jacobsen GK. Preliminary experience with a new method of endoscopic transbronchial real time ultrasound guided biopsy for diagnosis of mediastinal and hilar lesions. Thorax 2003; 58: 1083 - 1086

${ }^{25}$ Rintoul RC, Skwarski K, Hill AT et al. Endoscopic and endobronchial ultrasound real-time fine-needle aspiration for staging of the mediastinum in lung cancer. Chest 2004; 126: 2020-2022

${ }^{26}$ Yasufuku K, Chiyo M, Sekine Y et al. Real-time endobronchial ultrasound-guided transbronchial needle aspiration of mediastinal and hilar lymph nodes. Chest 2004; 126: $122-128$

${ }^{27}$ Rintoul RC, Skwarski K, Murchison JT et al. Endobronchial and endoscopic ultrasound-guided real-time fine needle aspiration for mediastinal staging. Eur Resp J 2005; 25: 416-421

${ }^{28}$ Yasufuku K, Chiyo M, Koh E et al. Endobronchial ultrasound guided transbronchial needle aspiration for staging of lung cancer. Lung Cancer 2005; 50: 347-354

${ }^{29}$ Vilmann P, Krasnik M, Larsen SS et al. Transesophageal endoscopic ultrasound-guided fine-needle aspiration (EUS-FNA) and endobronchial ultrasound-guided transbronchial needle aspiration (EBUS-TBNA) biopsy: a combined approach in the evaluation of mediastinal lesions. Endoscopy 2005; 37: $833-839$

${ }^{30}$ Mateu-Navarro M, Rami-Porta R, Bastus-Piulats R et al. Remediastinoscopy after induction chemotherapy in non-small cell lung cancer. Ann Thorac Surg 2000; 70: 391 - 395

${ }^{31}$ Van Schil P, Schoot van der J, Poniewierski J et al. Remediastinoscopy after neoadjuvant chemotherapy for non-small cell lung cancer. Lung Cancer 2002; 37: $281-285$

32 Pitz C, Mass K, Swieten H et al. Surgery as part of combined modality treatment in stage IIIB non-small cell lung cancer. Ann Thorac Surg 2002; 74: $164-169$

${ }^{33}$ Annema JT, Veselic M, Versteegh MIM et al. Mediastinal restagin$\mathrm{g}$ :EUS-FNA offers a new perspective. Lung Cancer 2003; 42: 311 - 318

${ }^{34}$ Rusch V. Mediastinoscopy: an endangered species? J Clin Onc 2005; 23: $8283-8285$ 